\title{
Identification and genetic characterization of Toxoplasma gondii in free-ranging bristle-spined porcupine (Chaetomys subspinosus), a threatened arboreal mammal from the Brazilian Atlantic Forest
}

\author{
Rodrigo Alves Bezerra', Gastón Andrés Fernandez Giné ${ }^{1}$, Bianca Mendes Maciel ${ }^{1}$, Fernanda Amato Gaiotto ${ }^{1}$ \\ and George Rêgo Albuquerque $2^{2^{*}}$
}

\begin{abstract}
Background: Strains of Toxoplasma gondii in Brazil have high genetic diversity compared to North America and Europe. The bristle-spined porcupine, Chaetomys subspinosus, is often subject to hunting for human food, but it is not known whether it can be a reservoir of this parasite. The aim of this study was to verify the occurrence of T. gondii in C. subspinosus from southern Bahia, Brazil, and genetically characterize and compare the strains found with those isolated in previous studies of the same region to quantify their genetic diversity by multilocus PCR-RFLP and PCR sequencing.
\end{abstract}

Findings: Twelve free-ranging C. subspinosus captured in forest fragments of the Una Biological Reserve and adjacent areas were evaluated. Three isolates of $T$. gondii (TgCsBr01-03) were detected. Two different genotypes were identified by applying multilocus PCR-RFLP with six molecular markers (SAG1, SAG2, SAG3, C22-8, PK1, and Apico). The isolates $\mathrm{TgCsBrO2}$ and TgCsBr03 were indistinguishable by this technique. However, the three isolates differed from all the reference strains and from the samples from the same region. Nevertheless, when the six genetic markers were used in multilocus PCR sequencing, all three isolates of $T$. gondii were different. The phylogenetic analysis revealed a greater genetic distance for TgCsBr01, which was closer to isolates from pigs from the same region, while TgCsBr02-03 was classified in the same lineage and was closer to isolates from sheep from this region.

Conclusions: All the isolates differed from the clonal genotypes of types I, II, and III using both genotyping techniques. Keywords: Genetic diversity, Population structure, Genotype, Sequencing, Toxoplasmosis

\section{Background}

Toxoplasma gondii is an obligate intracellular parasite that infects humans and a variety of warm-blooded animals as the intermediate hosts, with felines as the definitive hosts. T. gondii infections are widely prevalent in humans and animals throughout the world [1].

Molecular studies using PCR-RFLP and microsatellite analysis on isolates of T. gondii in North Africa, Europe, and North America classified the genetic lineages into

\footnotetext{
* Correspondence: gralbu@uesc.br

${ }^{2}$ Departamento de Ciências Agrárias e Ambientais, Universidade Estadual de Santa Cruz - UESC, Rodovia Jorge Amado, Km 16, Salobrinho, Ilhéus, BA 45662-900, Brazil

Full list of author information is available at the end of the article
}

three types, designated types I, II, III. However, the use of new molecular markers and the study of isolates from South America, especially Brazil, have shown that $T$. gondii has higher genetic variability [2-4].

Usually, wild animals are reservoirs of $T$. gondii, and the consumption of their raw or undercooked meat by humans may transport this protozoan $[5,6]$. In the Neotropics, consumption of bushmeat from hunting activity by local people is common [7]. In addition to increasing the risk of human contamination by zoonoses, hunting in this biome subjects several species of mammals to direct risk of extinction [8]. This is the case of the bristle-spined porcupine, Chaetomys subspinosus, a rodent species (of the family Erethizontidae) that is an 
arboreal folivore [9] of medium size [7] and endemic to the Atlantic Forest, whose populations are subject to strong hunting pressure throughout its distribution area, mainly for human consumption [10].

The aim of this study was to verify the occurrence of T. gondii in C. subspinosus from southern Bahia, northeastern Brazil, as well as to genetically characterize and compare the strains found with those isolated in previous studies of the same region to verify their genetic diversity through multilocus PCR-RFLP and PCR sequencing techniques.

\section{Findings \\ Methods \\ Collection of samples of biological material}

Ethical approval Experimental samples (brain and blood) [11] were collected from 12 free-ranging adult bristlespined porcupines from the Una Biological Reserve and adjacent areas, located in southern Bahia, Brazil, from January to November 2013. Of these 12 animals, 8 were captured, sedated [9], the other 4 were found dead by researchers or local residents. All the procedures were performed under the legal approval and consent of the Brazilian Federal Authority (ICMBio, license number: 25184-1; 23468-2 and 27021-1). The proposed study was approved by the ethics committee (CEUA-UESC 024/13).

\section{Molecular diagnosis and genetic characterization of Toxoplasma gondii}

DNA extraction DNA from blood (8 animals) and brain (4 animals) samples was extracted using the commercial kit Easy-DNA ${ }^{\mathrm{TM}}$ (Invitrogen). Tachyzoites of the RH strain were diluted at $\left(10^{7} / \mathrm{mL}\right)$ and homogenized, for use as a positive control. DNA was extracted and performed according to Bezerra et al. [3]. Samples stored at $-20^{\circ} \mathrm{C}$.

Diagnosis by PCR $T$. gondii was detected by polymerase chain reaction (PCR) which amplified a fragment of 529 bp utilizing the primers Tox4 Forward (CGCTGCAGGGAG GAAGACGAAAGTTG) and Tox5 reverse (CGCTGCA GACACAGTGCATCTGGATT) [12].

PCR-RFLP for genetic characterization The genotypes of $T$. gondii isolated were determined by means of multilocus PCR-RFLP with six genetic markers: SAG1, SAG2, SAG3, c22-8, PK1, and Apico. The amplification reactions were performed according to Bezerra et al. [3]. The digestions were carried out according to $\mathrm{Su}$ et al. [13]. The patterns of the DNA bands of the samples were compared with the genotypes deposited in ToxoDB (http://toxodb.org/toxo/).

DNA sequencing The products from nested PCR were purified using PureLink ${ }^{\mathrm{TM}}$ (Invitrogen) and sequenced for six genetic markers (SAG1, SAG2, SAG3, c22-8, PK1, and Apico) using the automatic sequencer ABI-PRISM 3100 Genetic Analyzer (Applied Biosystems). As positive controls, nested PCR products of RH (type I), PTG (type II), and CTG strains (type III) were sequenced. Nucleotide sequences determined in this study were assembled in contigs using CAP3. The sequences of T. gondii were aligned with ClustalW (version 1.83; [14]), manually corrected using BioEdit Sequence Alignment Editor, and compared with 10 reference sequences of $T$. gondii available at NCBI (http://www.ncbi.nlm.nih.gov/bioproject/). These strains were GT1 (PRJNA16727), ME49 (PRJNA28893), VEG (PRJNA19097), FOU (PRJNA61561), MAS (PRJNA 61545), VAND (PRJNA60839), RUB (PRJNA61119), p89 (PRJNA61547), TgCATBr5 (PRJNA61551), and TgCATBr9 (PRJNA61549). For the Apico marker, the sequences were aligned with the $T$. gondii apicoplast complete genome (U87145.2). All the sequences were compared with sequences available at ToxoDB.

Phylogenetic and statistical analysis The phylogenetic analysis was performed using MEGA version 6 by means of the neighbor-joining algorithm, and the distances were computed using the Tajima-Nei method. The stability of branches was assessed after bootstrapping with 500 replicates. To verify the distance between samples of the same geographical origin, eight $T$. gondii isolated from pigs from southern Bahia (TgPgBr06, TgPgBr08, TgPgBr09, TgPgBr11, TgPgBr12, TgPgBr13, TgPgBr15, and TgPgBr16) [3] and three T. gondii isolated from sheep from southern Bahia (\#54, \#124, and \#127) [4] were included. Tajima's test of neutrality [15] was used to compare the number of segregating sites with the nucleotide diversity of the DNA sequences.

\section{Results}

Genetic characterization by multilocus PCR-RFLP

Three $(25 \%)$ animals were positive for $T$. gondii based on the biological samples. The positive samples were from three different and adjacent forest fragments within the Una Biological Reserve: TgCsBr01, TgCsBr02 and TgCsBr03. The greatest distance between the positive records was approximately $6 \mathrm{~km}$. The application of PCRRFLP with six genetic markers (SAG1, SAG2, SAG3, c22-8, PK1, and Apico) revealed two genotype groups in the three isolates (Table 1).

\section{Genetic characterization by multilocus PCR sequencing}

A total of 1,604 positions were used in the alignment to calculate the evolutionary rate among the concatenated sequences of the samples TgCsBr01, TgCsBr02, and TgCsBr03, which was indicated through Tajima's relative rate test. There were 1,461 identical sites and no divergent sites between the three samples. The null hypothesis of 
Table 1 Multi-locus genotypes of Brazilian Toxoplasma gondii isolates by PCR-RFLP

\begin{tabular}{|c|c|c|c|c|c|c|c|}
\hline \multirow[b]{2}{*}{ T. gondii isolates } & \multicolumn{6}{|c|}{ Genetic markers } & \multirow[b]{2}{*}{ Reference } \\
\hline & $\overline{S A G 1}$ & SAG2 & SAG3 & $\mathrm{c} 22-8$ & PK1 & Apico & \\
\hline \multicolumn{8}{|l|}{ From C. subspinosus in Brazil } \\
\hline $\operatorname{TgCsBr} 01$ & । & I & III & । & III & III & \multirow[t]{3}{*}{ This study } \\
\hline TgCsBr 02 & I & । & $\|$ & III & I & III & \\
\hline $\mathrm{TgCsBr} 03$ & I & I & III & III & I & III & \\
\hline \multicolumn{8}{|l|}{ From reference strains (clonal types I, II and III) } \\
\hline RH88 (I) & I & । & । & । & I & I & \multirow[t]{3}{*}{ Dubey et al. [16] } \\
\hline CTg (II) & $\|/\| \|$ & $\|$ & $\|$ & $\|$ & $\|$ & $\|$ & \\
\hline PTg (III) & $\|/\| \|$ & III & III & III & III & III & \\
\hline \multicolumn{8}{|l|}{ From Brazilian genotypes } \\
\hline $\mathrm{Brl}$ & । & । & III & $u-1$ & I & । & \multirow[t]{4}{*}{ Pena et al. [2] Dubey et al. [16] } \\
\hline Brll & I & $\|$ & III & । & $\|$ & III & \\
\hline Brlll & I & III & III & $\|$ & III & III & \\
\hline BrIV & $u-1$ & । & III & $u-1$ & III & । & \\
\hline \multicolumn{8}{|l|}{ From the same geographic origin of this study - sheep } \\
\hline \#54 & I & I & । & III & $u-1$ & III & \multirow[t]{3}{*}{ Maciel et al. [4] } \\
\hline \#124 & I & I & I & III & $u-1$ & III & \\
\hline \#127 & I & 1 & I & III & $\mathrm{u}-1$ & III & \\
\hline \multicolumn{8}{|l|}{ From the same geographic origin of this study - pigs } \\
\hline TgPgBr06/TgPgBr08/TgPgBr11//gPgBr12/TgPgBr14/TgPgBr15 & । & । & III & । & I & III & \multirow[t]{6}{*}{ Bezerra et al. [3] } \\
\hline $\mathrm{TgPgBr} 7$ & 1 & 1 & III & $\mathrm{u}-1$ & ND & III & \\
\hline $\operatorname{TgPgBr} 9$ & 1 & I & III & I & I & III & \\
\hline TgPgBr 10 & $u-1$ & I & III & III & I & III & \\
\hline TgPgBr 13 & 1 & I & III & I & $\mathrm{u}-1$ & III & \\
\hline $\mathrm{TgPgBr} 16$ & I & 1 & III & I & I & III & \\
\hline
\end{tabular}

$u-1$ atypical alleles, ND not determined

equal rates among the lineages was rejected $(P<0.05)$, and one of the three samples (TgCsBr01) was considered to be from a different lineage.

The samples differed from the 10 strains of $T$. gondii and the apicoplast genome, presenting a mean of 120.6 DNA polymorphisms (5.2\%), including insertion, deletion, transition, and transversion, distributed over the different genetic markers (Table 2).

Through Tajima's $D$ test, which measures the distribution of allele frequency based on the data of nucleotide sequences, 1,870 bases could be aligned, showing 388 segregating sites. The analysis showed a positive result (0.372), which was indicative of a low number of lowfrequency polymorphism among all the strains of $T$. gondii used in this analysis.

\section{Phylogenetic analysis}

The phylogenetic analysis of the DNA sequences by the neighbor-joining method demonstrated that $\mathrm{TgCsBr} 02$ and $\mathrm{TgCsBr} 03$ were grouped in a group distinct from $\mathrm{TgCsBr} 01$. $\mathrm{TgCsBr} 02$ and $\mathrm{TgCsBr} 03$ were considered a sibling group, defined by an apomorphy of $99 \%$ of the bootstrap replicates. TgCsBr01 presented the largest branch length, which was proportional to the amount of polymorphisms. The isolates $\mathrm{TgCsBr} 02$ and $\mathrm{TgCsBr} 03$ were the closest to the reference strains GT1 (type I), ME49 (type II), and VEG strain (type III), but were grouped in a distinct group with $15 \%$ of the bootstrap replicates (Fig. 1).

\section{Discussion}

This was the first study that identified and genetically characterized T. gondii from C. subspinosus. None of the samples were classified as a clonal genotype of type I, II, or III (Table 1) or as a main Brazilian clonal genotype (BRI, BRII, BrIII, and BRIV) defined by Dubey et al. [16] and Pena et al. [2]. The samples were also genetically distinct from genotypes of $T$. gondii previously isolated from pigs and sheep of the same geographic region of Brazil (southern Bahia), as described by Bezerra et al. [3] and Maciel et al. [4] (Table 1). Moreover, the samples did not combine with any genotype deposited in ToxoDB. 
Table 2 Genotyping by PCR-RFLP and number of polymorphisms at six genetic loci detected by PCR sequencing

\begin{tabular}{|c|c|c|c|c|c|c|c|}
\hline \multirow[b]{2}{*}{ Isolate } & \multirow[b]{2}{*}{$\begin{array}{l}\text { Genotype } \\
\text { PCR-RFLP }\end{array}$} & \multicolumn{4}{|c|}{ No. of polymorphisms detected by sequencing } & \multirow[b]{2}{*}{$\begin{array}{l}\text { Sequence with the highest-scoring } \\
\text { segment pairs in ToxoDB }\end{array}$} & \multirow[b]{2}{*}{$\begin{array}{l}\text { Identity (\%); } \\
\text { Expected value }\end{array}$} \\
\hline & & Indel & Ts & TV & Total & & \\
\hline \multicolumn{8}{|c|}{ Marker SAG1 (225 bp) - Chromosome VIII Coding function: Surface antigen gene } \\
\hline TgCsBr01 & । & 0 & 0 & 0 & $0(0.0 \%)$ & TgUgCh83 (EF534734.1) & $100 ; 4 \mathrm{e}-113$ \\
\hline $\mathrm{TgCsBr02}$ & । & 0 & 0 & 0 & $0(0.0 \%)$ & TgUgCh83 (EF534734.1) & $100 ; 4 \mathrm{e}-113$ \\
\hline TgCsBrO3 & । & 0 & 0 & 0 & $0(0.0 \%)$ & TgUgCh83 (EF534734.1) & $100 ; 4 \mathrm{e}-113$ \\
\hline
\end{tabular}

Marker SAG2 (385 bp) - Chromosome VIII Coding function: Surface antigen gene

$\begin{array}{llllllll}\text { TgCsBr01 } & \text { I } & 1 & 2 & 3 & 6(1.5 \%) & \text { TgCkNg1 (EU650330.1) } & 99 ; 0.0 \\ \text { TgCsBr02 } & \text { I } & 1 & 2 & 3 & 6(1.5 \%) & \text { TgCkNg1 (EU650330.1) } & 99 ; 0.0 \\ \text { TgCsBr03 } & \text { 1 } & 7 & 4 & 3 & 14(3.6 \%) & \text { TgCkNg1 (EU650330.1) } & 97 ; 0.0\end{array}$

Marker SAG3 (115 bp) - Chromosome XII Coding function: Surface antigen gene

$\begin{array}{llllllll}\text { TgCsBr01 } & \text { II } & 0 & 0 & 0 & 0(0.0 \%) & \text { Tg strain CTG (JX218227.1) } & 100 ; 3 e-52 \\ \text { TgCsBr02 } & \text { III } & 1 & 1 & 1 & 3(2.6 \%) & \text { Tg strain CTG (JX218227.1) } & 99: 2 \mathrm{e}-49 \\ \text { TgCsBr03 } & \text { III } & 2 & 0 & 0 & 2(1.7 \%) & \text { Tg strain CTG (JX218227.1) } & 98: 5 \mathrm{e}-44\end{array}$

Marker c22-8 (485 bp) - Chromosome Ib Coding function: unknown "conserved hypothetical protein"

$\begin{array}{llllllll}\text { TgCsBr01 } & \text { I } & 47 & 64 & 72 & 183(37.7 \%) & \text { TgCatBr5 (EU258488.1) } & 90 ; 1 \text { - } 94 \\ \text { TgCsBr02 } & \text { III } & 2 & 3 & 0 & 5(1.0 \%) & \text { Tg PTG (EU258476.1) } & 100 ; 0.0 \\ \text { TgCsBr03 } & \text { III } & 3 & 1 & 1 & 5(1.0 \%) & \text { Tg PTG (EU258476.1) } & 98 ; 0.0\end{array}$

Marker PK1 (660 bp) - Chromosome VI Coding function: Protein serine/threonine kinase gene

\begin{tabular}{|c|c|c|c|c|c|c|c|}
\hline TgCsBr01 & III & 5 & 0 & 1 & $6(0.9 \%)$ & TgCkNg1 (EU650328.1) & $99 ; 0.0$ \\
\hline TgCsBrO2 & I & 5 & 0 & 1 & $6(0.9 \%)$ & TgCkNg1 (EU650328.1) & $99 ; 0.0$ \\
\hline $\mathrm{TgCsBrO3}$ & I & 0 & 0 & 1 & $1(0.1 \%)$ & TgCkNg1 (EU650328.1) & $99 ; 0.0$ \\
\hline \multicolumn{8}{|c|}{ Marker Apico ${ }^{a}$ (461 bp) - Apicoplast chromosome } \\
\hline TgCsBr01 & III & 1 & 0 & 2 & $3(0.6 \%)$ & T. gondii Apicoplast, comp. genome (U87145.2) & $99 ; 0.0$ \\
\hline TgCsBr02 & III & 42 & 27 & 52 & $121(26.2 \%)$ & T. gondii Apicoplast, comp. genome (U87145.2) & $95 ; 5 e-30$ \\
\hline $\mathrm{TgCsBrO3}$ & III & 1 & 0 & 0 & 1 (0.2 \%) & T. gondii Apicoplast, comp. genome (U87145.2) & $99 ; 0.0$ \\
\hline
\end{tabular}

Total of polymorphisms at six different genetic loci detected by PCR sequencing of $T$. gondii isolates ${ }^{b}$

Total of polymorphisms (\%)

\begin{tabular}{|c|c|c|c|c|c|c|c|}
\hline Isolate & Genotype PCR-RFLP & Indel & Ts & TV & Total & Tajima's relative rate test ${ }^{c}$ & $\begin{array}{l}\text { Tajima's } D \\
\text { neutrality test }^{d}\end{array}$ \\
\hline TgCsBr01 & Atypical & 54 & 66 & 78 & $198(8.5 \%)$ & $u=137$ & \\
\hline TgCsBrO2 & Atypical & 51 & 33 & 57 & $141(6.0 \%)$ & $u=2$ & \\
\hline TgCsBrO3 & Atypical & 13 & 5 & 5 & $23(1.0 \%)$ & $u=4$ & \\
\hline average $b$ & etween samples & 39.3 & 34.6 & 46.6 & $120.6(5.2 \%)$ & $P=0.00000$ & $D=0.372232$ \\
\hline
\end{tabular}

${ }^{\mathrm{a}}$ The sequences were aligned with the $T$. gondii apicoplast complete genome

${ }^{\mathrm{b}}$ The number of insertions and deletions (Indel), transitions (Ts) and transversions (Tv) were calculated comparing the sequence of each isolate with the pattern obtained from GT1, ME49, VEG, TgCATBr5, TgCATBr9, FOU, RUB, VAND, p89, MAS, TgPgBr06, TgPgBr07, TgPgBr08, TgPgBr09, TgPgBr10, TgPgBr11, TgPgBr12, $\mathrm{TgPgBr} 13, \mathrm{TgPgBr} 14, \mathrm{TgPgBr} 15, \mathrm{TgPgBr} 16,54,124$ and 127 reference strains. The size of each amplicon means the number of base pairs that matched in all samples after the multiple alignment

'The equality of evolutionary rates between the sequences $\mathrm{TgCsBr01}, \mathrm{TgCsBr02}$ and $\mathrm{TgCsBr03.} \mathrm{"u"} \mathrm{means} \mathrm{unique} \mathrm{differences} \mathrm{in} \mathrm{each} \mathrm{sequence.} \mathrm{All} \mathrm{positions}$ containing gaps and missing data were eliminated. There were a total of 1604 positions with 1461 identical sites in all three sequences and 0 divergent sites between all three sequences. A $P$-value less than 0.05 is often used to reject the null hypothesis of equal rates between lineages ${ }^{\mathrm{d}}$ The analysis involved 27 multi-locus nucleotide sequences (GT1, ME49, VEG, TgCATBr5, TgCATBr9, FOU, RUB, VAND, p89, MAS, TgPgBr06, TgPgBr07, TgPgBr08, TgPgBr09, TgPgBr10, TgPgBr11, TgPgBr12, TgPgBr13, TgPgBr14, TgPgBr15, TgPgBr16, 54, 124, 127, TgCsBr01, TgCsBr02, TgCsBr03). All positions containing gaps and missing data were eliminated. There were a total of 1870 bases aligned with 388 segregating sites. A negative Tajima's $D$ indicates an excess of low-frequency polymorphisms. Evolutionary analyses were conducted in MEGA6

However, the phylogram determined by the analysis of the chromosomal genetic marker sequence revealed that the isolates $\mathrm{TgCsBr} 02$ and $\mathrm{TgCsBr} 03$ were closer to the ovine strains of the same area of study (\#54 and \#127). $\mathrm{TgCsBr} 01$ shared a monophyletic group in $99 \%$ of the replicates with swine isolates from the same geographical 


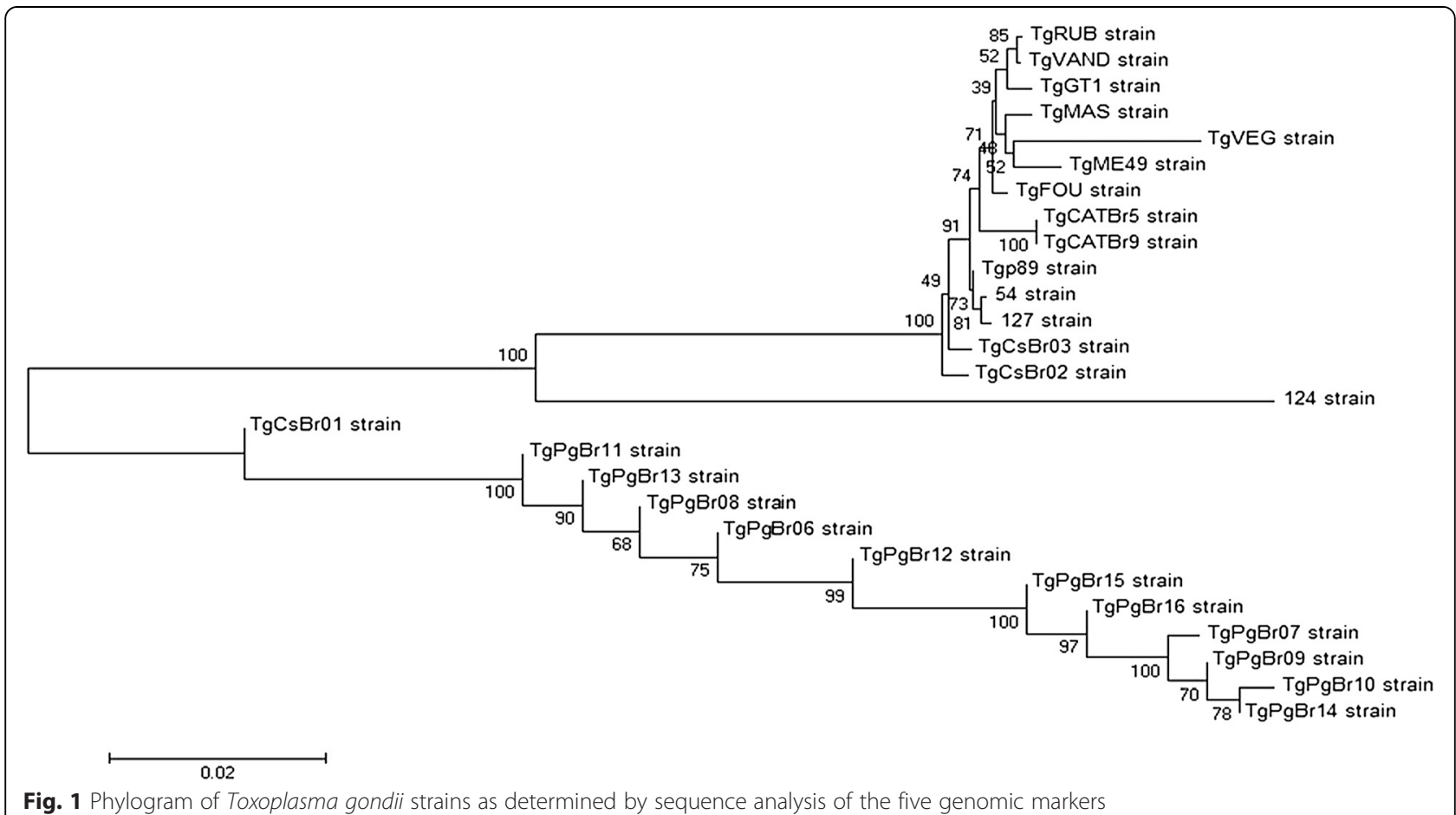

region (TgPgBr06, TgPgBr07, TgPgBr08, TgPgBr09, TgPgBr10, TgPgBr11, TgPgBr12, TgPgBr13, TgPgBr14, TgPgBr15, and $\mathrm{TgPgBr} 16)$, which suggests that it may belong to a close lineage that inhabits this region. However, the genetic characterization of the parasite carried out by means of multilocus PCR-RFLP and DNA sequencing techniques indicated a high genetic diversity of the parasite in the region (Tables 1 and 2; Fig. 1).

The genetic characterization by PCR-RFLP revealed two genotypes in the three strains. Nevertheless, it was verified that the isolates of $C$. subspinosus possessed many of the same alleles present in other Brazilian genotypes, although the alleles had distinct segregation among the loci examined, thereby characterizing it as a new genotype. The multilocus PCR sequencing indicated that the three isolates from the C. subspinosus samples were distinct, and the genetic variability between the samples was enough to classify them into different genotypes in the phylogenetic analysis. This was indicated by Tajima's relative rate test, which calculated the equality of evolutionary rates between the concatenated sequences generated by multilocus PCR sequencing (Fig. 1).

The most polymorphic genetic markers were c22-8 and Apico, with alignments presenting 90 and $95 \%$ identity with the TgCatBr5 and apicoplast genomes, respectively (Table 2). Despite this high degree of polymorphism, the samples were grouped in the clonal genotype type III by the PCR-RFLP technique (Table 1 ).
These regions may be considered effective to distinguish isolates of clonal types I, II, and III, though this complicates the grouping of Brazilian isolates.

Few studies have genetically characterized $T$. gondii in wild mammals, and most research in Brazil is related to serological techniques in these animals $[5,6,17,18]$. Most isolates of $T$. gondii genotyped in Brazil are from domestic animals, including chickens, cats, dogs, sheep, goats and pigs. The studies that have genetically characterized isolates of $T$. gondii from wild animals have also reported a high genetic diversity and have contributed to the elucidation of the Brazilian genotype network [1921]. Silva et al. [6], upon detecting T. gondii in armadillos in Brazil, claimed that these wild animals can be a source of transmission of the parasite to humans, mainly due to the rural people's habit of eating the meat of these animals.

\section{Conclusions}

Little is known about the genetic variability of isolates of T. gondii in wild mammals in Brazil. Knowing the genetic similarities or differences of $T$. gondii between different animal populations is necessary to understand the transmission of the parasite.

\section{Competing interests}

The authors declare that they have no competing interests. 


\section{Authors' contributions}

$R A B$ organized the study, performed the experiments and wrote the manuscript. GAFG significantly contributed to the sample collection. RAB and BMM performed molecular analysis; FAG arranged, designed and supervised the molecular studies. GRA designed the experiments. GAFG, FAG BMM and GRA helped in study design, study implementation and manuscript revision. All authors read and approved the manuscript.

\section{Acknowledgments}

The authors thank the Conselho Nacional de Desenvolvimento Científico e Tecnológico (CNPq) through the CASADINHO/PROCAD (Process 552198/ 2011-0) and the Coordenação de Aperfeiçoamento de Pessoal de Nível Superior (CAPES) (Process AUX-PE-Parasitologia - 1267/2011) for financial support.

\section{Author details}

${ }^{1}$ Departamento de Ciências Biológicas, Universidade Estadual de Santa Cruz UESC, Rodovia Jorge Amado, Km 16, Salobrinho, Ilhéus, BA 45662-900, Brazil. ${ }^{2}$ Departamento de Ciências Agrárias e Ambientais, Universidade Estadual de Santa Cruz - UESC, Rodovia Jorge Amado, Km 16, Salobrinho, Ilhéus, BA 45662-900, Brazil.

Received: 9 February 2015 Accepted: 4 May 2015

Published online: 17 May 2015

\section{References}

1. Tenter AM, Heckeroth AR, Weiss LM. Toxoplasma gondii: from animals to humans. Int J Parasitol. 2000;30:1217-58.

2. Pena HFJ, Gennari SM, Dubey JP, Su C. Population structure and mousevirulence of Toxoplasma gondii in Brazil. Int J Parasitol. 2008;38:561-9.

3. Bezerra RA, Carvalho FS, Guimarães LA, Rocha DS, Maciel BM, Wenceslau AA, et al. Genetic characterization of Toxoplasma gondii isolates from pigs intended for human consumption in Brazil. Vet Parasitol. 2012;189:153-61.

4. Maciel BM, Moura RL, Carvalho FS, Costa EA, Albuquerque GR. Identification and genetic characterization of a new Brazilian genotype of Toxoplasma gondii from sheep intended for human consumption. Parasitol Int. 2014;63:567-70.

5. Silva AV, Bosco SMG, Langoni H, Bagagli E. Study of toxoplasma infection in Brazilian wild mammals: serological evidence in Dasypus novemcinctus Linnaeus, 1758 and Euphractus sexcinctus Wagler, 1830. Vet Parasitol. 2006;135:81-3.

6. Silva RC, Zetun CB, Bosco SMG, Bagagli E, Rosa PS, Langoni H. Toxoplasma gondii and Leptospira spp. Infection in free-ranging armadillos. Vet Parasitol. 2008;157:291-3.

7. Eisenberg JF, Redford KH. Mammals of the Neotropics. The Central Neotropics. Ecuador, Peru, Bolivia, Brazil. 3rd ed. Chicago: The University of Chicago Press; 1999.

8. Cullen L, Bodmer RE, Pádua CV. Effects of hunting in habitat fragments of the Atlantic Forest. Brazil Biol Conserv. 2000;95:49-56

9. Giné GAF, Duarte JMB, Faria D. Feeding ecology of a selective folivore: the case of the thin-spined porcupine (Chaetomys subspinosus) in the Atlantic Forest. J Mammal. 2010:91:931-41.

10. Oliver WLR, Santos IB. Threatened endemic mammals of the Atlantic forest region of south-eastern Brazil. Wildlife Preservation Trust Special Scientific Report. 1991.

11. Mancianti F, Nardoni S, Papini R, Mugnaini L, Martini M, Altomonte I, et al. Detection and genotyping of Toxoplasma gondii DNA in the blood and milk of naturally infected donkeys (Equus asinus). Parasit Vectors. 2014;7:165.

12. Homan WL, Vercammen M, De Braekeleer J. Identification of a 200-300 fold repetitive 529 bp DNA fragment in Toxoplasma gondii, and its use for diagnostic and quantitative PCR. Int J Parasitol. 2000;30:69-75.

13. Su C, Shwab EK, Zhou P, Zhu XQ, Dubey JP. Moving towards an integrated approach to molecular detection and identification of Toxoplasma gondii. Parasitology. 2010;137:1-11.

14. Thompson JD, Higgins DG, Gibson TJ. CLUSTAL W: improving the sensitivity of progressive multiple sequence alignment through sequence weighting, position specific gap penalties and weight matrix choice. Nucleic Acids Res. 1994;22:4673-80

15. Tajima F. Statistical methods to test for nucleotide mutation hypothesis by DNA polymorphism. Genetics. 1989;123:585-95.
16. Dubey JP, Velmurugan GV, Chockalingam A, Pena HFJ, Oliveira LN, Leifer CA, et al. Genetic diversity of Toxoplasma gondii isolates from chickens from Brazil. Vet Parasitol. 2008;157:299-305.

17. Silva RC, Machado GP, Cruvinel TMA, Cruvinel CA, Langoni H. Frequency of Toxoplasma gondii antibodies in tufted capuchin monkeys (Cebus apella nigritus) from an ecological station in the State of São Paulo. Brazil Pesq Vet Bras. 2013;33:251-3.

18. Vitaliano SN, Soares HS, Pena HFJ, Dubey JP, Gennari SM. Serologic evidence of Toxoplasma gondii infection in wild birds and mammals from southeast Brazil. J Zoo Wildlife Med. 2014;45:197-9.

19. Yai LEO, Ragozo AMA, Soares RM, Pena HFJ, Su C, Gennari SM. Genetic diversity among capybara (Hydrochaeris hydrochaeris) isolates of Toxoplasma gondii from Brazil. Vet Parasitol. 2009;162:332-7.

20. Pena HFJ, Marvulo MFV, Horta MC, Silva MA, Silva JCR, Siqueirae DB, et al. Isolation and genetic characterisation of Toxoplasma gondii from a redhanded howler monkey (Alouatta belzebul), a jaguarundi (Puma yagouaroundi), and a black-eared opossum (Didelphis aurita) from Brazil. Vet Parasitol. 2011;175:377-81.

21. Canón-Franco WA, Araújo FAP, López-Orozco N, Jardim MMA, Keide LB, Dalla-Rosa C, et al. Toxoplasma gondii in free-ranging wild small felids from Brazil: Molecular detection and genotypic characterization. Vet Parasitol. 2013;197:462-9.

\section{Submit your next manuscript to BioMed Central and take full advantage of:}

- Convenient online submission

- Thorough peer review

- No space constraints or color figure charges

- Immediate publication on acceptance

- Inclusion in PubMed, CAS, Scopus and Google Scholar

- Research which is freely available for redistribution

Submit your manuscript at www.biomedcentral.com/submit
C Biomed Central 